\title{
The DSM 5 and the Istanbul Protocol: Diagnosis of psychological sequels of torture
}

\author{
Thomas Wenzel, MD*,**, Andreas Frewer***, Siroos Mirzaei****
}

\begin{abstract}
The Manual on Effective Investigation and Documentation of Torture and Other Cruel, Inhuman or Degrading Treatment or Punishment, commonly known as the Istanbul Protocol, is an interdisciplinary standard supported by, among others, the United Nations and the World Medical Association. It aims at aiding the fight against torture by giving clear guidelines to ensure better and more effective assessment of physical and psychological sequels. Mental health is a key aspect of diagnostical assessment and documentation due to the severe and frequently long-lasting impact of torture that often lasts longer than physical sequels. The inclusion of psychological aspects and a psychiatric diagnosis is to be treated as an important obligatory. Care must be taken to avoid common pitfalls. The new and substantial revisions in the frequently used but also criticised Diagnostical and Statistical Manual (DSM) reflect challenges and opportunities in a comprehensive approach to the documentation of torture.
\end{abstract}

\footnotetext{
^) Medical University of Vienna, Department of Social Psychiatry

${ }^{\star}$ ) World Psychiatric Association Section on Psychological Sequels to Torture and Persecution

$\star \star \star \star)$ University Erlangen-Nürnberg, Germany

$\star \star \star \star)$ Wilhelminenspital; Hemayat Treatment Centre, Vienna, Austria
}

Correspondence to: drthomaswenzel@web.de
Keywords: torture, PTSD, mental health, transcultural assessment

\section{The Istanbul Protocol and the assessment of victims of torture or inhuman and degrading treatment} The Manual on Effective Investigation and Documentation of Torture and Other Cruel, Inhuman or Degrading Treatment or Punishment, commonly known as the Istanbul Protocol (IP), is an interdisciplinary standard supported by the United Nations and endorsed by key umbrella healthcare organisations, including the World Medical Association (WMA), the World Council of Psychotherapy, the World Psychiatric Association (WPA) and the International Rehabilitation Council for Torture Victims (IRCT). The IP covers general medical, legal and psychological aspects of investigation and forensic diagnostic assessment. ${ }^{1-5}$ The development and international acceptance of this major tool reflects the understanding that a comprehensive forensic documentation which can stand up to the requirements of monitoring and legal procedures such as local or international courts is a crucial aspect in the prevention of torture and support of survivors. Due to its position as a recommended framework, it has been developed to provide general guidelines, but cannot offer a continuously updated handbook that includes all aspects of the rapidly developing related fields. For that reason, most of the IP is phrased in a general 
way that allows some flexibility, for example in the use of diagnostic categories. However, it specifically mentions the older DSM-IV version of the American Psychiatric Association's influential "Diagnostical and Statistical Manual" to document the impact of torture on mental health. The recent changes in the $5^{\text {th }}$ edition (DSM-5), ${ }^{6}$ regarding specific trauma- and stressor-related disorders and in transcultural factors appear to be of substantial importance for the assessment of survivors.

Mental health is a key aspect of diagnostical assessment and documentation because of

a) the severe impact that torture has on this aspect of health, that is frequently longer lasting than physical sequels;

b) the influence on overall medical assessment results that can lead to incorrect or incomplete evaluations, usually with a disadvantage for the survivor; and

c) the risk of undue stress or even retraumatisation through inadequate procedures.

Psychological aspects should according to the IP be included in all assessments, but care must be taken in reaching a diagnosis.

\section{Using a diagnosis in assessment of survivors of torture or inhuman and degrading treatment}

The clinical assessment of torture survivors poses numerous difficulties for the clinician and forensic expert from the diagnostic standpoint. While it is theoretically feasible to just describe findings without giving a final diagnosis in situations of doubt or ongoing differential diagnostic assessment, a diagnosis based on the World Health Organisation's International Classification of Diseases (ICD) $10^{\text {th }}$ revision ICD-10-CM, or alternatively a DSM diagnosis for mental health sequels is commonly expected.
If any of these systems is used, the most recent versions should also be used in forensic assessment, though country standards might require otherwise and a gradual transition might be needed. DSM in itself must be seen as a common but not an obvious choice as it is not the recommended standard of the World Health Organisation (WHO) and is limited to mental health. Due to its predominant use in many countries and in research, and the impact revisions usually have on both amendments of the ICD and on the perception of mental health problems in general, important changes should be considered in treatment and assessment of victims of torture. Transition is supported in DSM- 5 by listing comparable ICD-9-CM (Clinical Modification) and ICD-10-CM codes, and by keeping contact with the ICD-11 development team.

Furthermore, the dimensional models that have recently been required for example for some funded research have also been considered. The seemingly not quite reasonable use of "outdated" ICD-9-CM and ICD-10-CM codes in many countries answers to needs of healthcare contractors, but could lead to misunderstandings in forensic settings. It should in this context be considered that the DSM was originally developed as a statistical and research tool that requires a number of strict criteria to be fulfilled and would rather err on the sensitivity then on the specificity (avoiding a diagnosis, if in doubt). In the worst case, this could mean that a person suffering might be mistakenly understood as not being sick and not in need of treatment, or that the impact of torture might not be recognised if the model would be applied in a clinical or forensic setting.

However, changes in the recent DSM (version 5) could be seen as a significant improvement in spite of some reservations. 


\subsection{Standard instruments}

While a clinical interview by an experienced clinician is usually a sufficient and even potentially the "gold" standard also recommended by the IP, questionnaires, such as the Harvard Trauma Questionnaire ${ }^{7}$ or standardised schedules, including the Clinician-Administered PTSD Scale (CAPS $)^{8,9}$ are also used, especially in screening and to confirm a clinical diagnosis. The IP is rather careful in recommending testing and specific instruments, especially as a validation in regards to language and culture is frequently missing. The substantial revision of the PTSD criteria in DSM-5 will obviously require a revision and revalidation of all instruments. This will be a challenge, as validated versions of key instruments have even now not been published in most languages and might require substantial resources. The two instruments mentioned above have the advantage that they have already been translated in numerous languages and that they share the inclusion of most symptoms added in DSM- 5 such as shame and guilt feelings or dissociative symptoms.

\subsection{Taking care with a diagnosis}

The doubts as to the limitations of key concepts such as PTSD underline the need to use a diagnosis with care, and the IP gives the option of a descriptive approach, further confirming the need for a culturally sensitive assessment that is independent from standard diagnostic categories - a key point which we will cover later on. It might also be considered that a psychiatric diagnosis can be stigmatising in many cultures, though it may be required by the forensiclegal setting.

A special risk is the misunderstanding of the conclusion that "criteria for a disorder are not fulfilled", which could suggest a lack of credibility, contradict suffering from sequels, or imply that no torture was experienced. A general cautionary approach should include an explanation of this reservation to the frequently non-medical or legal authorities receiving the report. It should also make use of an extended diagnostic model such as the option of giving a diagnosis of "not otherwise specified" disorder (NOS) that can be used if not all criteria are fulfilled, but significant suffering is caused by a limited number of the required symptoms.

\subsection{Psychological aspects in the Istanbul Protocol}

Psychological sequelae are of especially high relevance ${ }^{10}$ due to several factors outlined also in the IP, as they are common, can be long-lasting or life-long, can be severely debilitating or can interact or interfere with concentration, attention and memory functions. ${ }^{11}$

All above aspects mentioned are of relevance for medical assessment that preserves evidence of torture and identifies treatment needs, but also for the legal professionals who have to consider key issues such as the possibility of memory disturbance interfering with testimony and narrative.

While many reactive patterns including PTSD can be seen as "normal reactions to an abnormal event", especially in a dimensional approach, they are relevant as evidence and as factors in an interview or examination. All professionals interacting with victims of torture need to avoid undue stress and suffering that can easily be caused by inadequate procedures in any interaction with the survivor and should be brought to the attention also of legal professionals.

Unspecific disorders such as depression 
are at least equally frequent as specific trauma related disorders, but the following summary will due to limitations of space focus on changes in the key areas of stress related and transcultural aspects. In the comprehensive assessment, however, common co-morbidity and the high prevalence of unspecific disorders, especially depression, somatoform and dissociative disorders ${ }^{11-14}$ should be considered.

\subsection{Relevant changes in the DSM-5}

2.4.1. General aspects of changes in the DSM-5 structure

The earlier 5-axis model of DSM-IV has been abandoned in favour of a more evidence based approach, integrating validated diagnostic instruments, especially the WHO Disability Assessment Schedule (WHODAS), ${ }^{15}$ which is an often neglected but potentially very relevant part of assessment and a "cross-symptom" approach that yields data on overlapping areas.

\subsubsection{Trauma- and Stressor-Related Disorders} DSM underlines causal links between events and specific stressful life events through both the inclusion of this special category and the use of $\mathrm{V}$ codes that are also used in ICD-10-CM and will be explained later in this article.

PTSD, especially in younger children (6 years or younger), is now defined by an extensive set of specific criteria that reflect the special posttraumatic stress patterns in this group, while the IP includes very limited guidelines for children. Still, children present a broad range of reactions that are further influenced by culture and not only limited to PTSD. ${ }^{16}$ Creative media can be considered in differential diagnosis.

\subsubsection{Posttraumatic Stress Disorder (PTSD)}

The often criticised DSM-5 Criterion A has been specially revised and no longer asks for a specific subjective reaction during the event. A further major change that might require modification in most present diagnostic instruments is the inclusion of a fourth cluster of symptoms (D: "persistent negative alterations in cognitions and mood") that reflects also the embedding of the earlier "complex" symptoms ("DESNOES”, "Complex PTSD”), including "social" emotions such as shame or guilt feelings and behaviour and withdrawal. They are common and characteristic for victims of torture. Dissociative symptoms are now included as an additional subcategory ("with depersonalisation" or "with derealisation"). Dissociation might interfere with concentration and memory and is therefore relevant in assessment and in evaluating a narrative of torture.

The discussion of late onset forms ("with delayed expression"), especially in forensic settings, is addressed by a flexible set of criteria that permit for early onset of some symptoms, with full criteria met at any point in time later on ("whenever"). Late onset has been a frequently challenged aspect in forensic assessment of severe trauma.

\subsubsection{Acute Stress Disorder}

Acute stress disorder might be important as an early warning sign for the immediate impact of inhuman or degrading treatment and torture.

\subsubsection{Adjustment Disorders}

Adjustment disorders have been "reconceptualised as a heterogeneous array of stressresponse syndromes that occur after exposure to a distressing (traumatic or non-traumatic) event". This diagnosis can be used as an alternative to denote a larger range of symptoms with a specific causal relationship to torture or other less severe events. 
Table 1: Posttraumatic Stress Disorder DSM-5 criteria

A. Exposure to actual or threatened death, serious injury, or sexual violence in one (or more) of the following ways:
1. Directly experiencing the traumatic event(s).

2. Witnessing, in person, the event(s) as it occurred to others.

3. Learning that the traumatic event(s) occurred to a close family member or close friend. In cases of actual or threatened death of a family member or friend, the event(s) must have been violent or accidental.

4. Experiencing repeated or extreme exposure to aversive details of the traumatic event(s) (e.g., first responders collecting human remains: police officers repeatedly exposed to details of child abuse).

B. Presence of one (or more) of the following intrusion symptoms associated with the traumatic event(s), beginning after the traumatic event(s) occurred:
1. Recurrent, involuntary, and intrusive distressing memories of the traumatic event(s).

Note: In children older than 6 years, repetitive play may occur in which themes or aspects of the traumatic event(s) are expressed.

2. Recurrent distressing dreams in which the content and/or affect of the dream are related to the traumatic event(s).

Note: In children, there may be frightening dreams without recognizable content.

3. Dissociative reactions (e.g., flashbacks) in which the individual feels or acts as if the traumatic event(s) were recurring. (Such reactions may occur on a continuum, with the most extreme expression being a complete loss of awareness of present surroundings.)

Note: In children, trauma-specific reenactment may occur in play. 
4. Intense or prolonged psychological distress at exposure to internal or external cues that symbolize or resemble an aspect of the traumatic event(s).

5. Marked physiological reactions to internal or external cues that symbolize or resemble an aspect of the traumatic event(s).

C. Persistent avoidance of stimuli associated with the traumatic event(s), beginning after the traumatic event(s) occurred, as evidenced by one or both of the following:
1. Avoidance of or efforts to avoid distressing memories, thoughts, or feelings about or closely associated with the traumatic event(s).

2. Avoidance of or efforts to avoid external reminders (people, places, conversations, activities, objects, situations) that arouse distressing memories, thoughts, or feelings about or closely associated with the traumatic event(s).

D. Negative alterations in cognitions and mood associated with the traumatic event(s), beginning or worsening after the traumatic event(s) occurred, as evidenced by two (or more) of the following:
1. Inability to remember an important aspect of the traumatic event(s) (typically due to dissociative amnesia and not to other factors such as head injury, alcohol, or drugs).

2. Persistent and exaggerated negative beliefs or expectations about oneself, others, or the world.

3. Persistent, distorted cognitions about the cause or consequences of the traumatic event(s) that lead the individual to blame himself/herself or others.

4. Persistent negative emotional state (e.g., fear, horror, anger, guilt, or shame).

5. Markedly diminished interest or participation in significant activities.

6. Feelings of detachment or estrangement from others.

7. Persistent inability to experience positive emotions (e.g., inability to experience happiness, satisfaction, or loving feelings). 
E. Marked alterations in arousal and reactivity 1 . Irritable behavior and angry outbursts associated with the traumatic event(s), (with little or no provocation) typically ex beginning or worsening after the traumatic event(s) occurred, as evidenced by two (or pressed as verbal or physical aggression toward people or objects. more) of the following:

2. Reckless or self-destructive behavior.

3. Hypervigilance.

4. Exaggerated startle response.

5. Problems with concentration.

6. Sleep disturbance (e.g., difficulty falling or staying asleep or restless sleep).

F. Duration of the disturbance (Criteria B, C, $\mathrm{D}$, and $\mathrm{E}$ ) is more than 1 month.

G. The disturbance causes clinically significant distress or impairment in social, occupational, or other important areas of functioning.

$\mathrm{H}$. The disturbance is not attributable to the physiological effects of a substance (e.g., medication, alcohol) or another medical condition.

\subsubsection{Other Specified Trauma- and Stressor- Related Disorder}

This can be seen as an important collective category for reactions that do not meet the full criteria for another stress related disorder, and includes "Cultural Concepts of Distress", to be noted as "Other cultural syndromes" as outlined below. Specific aspects are listed together with the diagnosis. The new frame provides an important diagnostic option, especially in a transcultural setting or complex assessment. It also avoids the commonly observed misunderstanding that not fulfilling PTSD criteria for example if a culture specific idiom of distress or not all symptoms are present could contradict traumatisation or even reported exposure to torture. An explanation of the concept might be necessary if used in a court or with a legal investigation team in addition to the diagnosis.

\subsubsection{Unspecified Trauma- and Stressor-Related Disorder}

These are "diagnosed in case of stress related symptoms when information is not available or should not be noted". The category can be used as a preliminary diagnosis.

\section{Other selected relevant disorders}

\subsubsection{Bereavement}

Complex bereavement disorder (as separate from a culture - "adequate" bereavement reaction) is a condition "under study", i.e. not yet officially a "full" category, and can be coded under "Other Specified Trauma- and 
Stressor-Related Disorders". Bereavement can be a special aspect of persecution and violence and should be considered in survivors of torture. It is also a common but neglected problem in survivors of war and other persecution.

\subsubsection{Brain trauma}

One of the potentially most overlooked problems. Even shoves, "mild" beatings or falls without obvious loss of consciousness which are common in torture can lead to persistent symptoms that can be misinterpreted as only PTSD related or missed, but have significant implications for assessment, proof of mistreatment, and treatment. ${ }^{17}$ Presence of major or mild neurocognitive disorders (NCDs) resulting from brain trauma should therefore be part of any assessment. DSM-5 offers support by using a table of neurocognitive strategies of assessment that are more concrete than, for example, the more general description in the IP.

\subsubsection{Transcultural aspects}

Unclear strategies in approaching cultural background are possibly the most controversial aspect of earlier DSM and ICD versions. The need of a culturally informed assessment is stressed in the IP.

DSM-5 has substantially extended this part which can be perceived as a significant improvement as it can be used to reflect the multiple cultures in a global society. It includes earlier descriptions of "culture bound syndromes" such as "Ataque de nervio", and "cultural formulations". It asks in general for assessment of "Cultural identity of the individual, cultural conceptualizations of distress, psychosocial stressors and cultural features of vulnerability and resilience, cultural features of the relationship between the individual and the clinician", and an "overall cultural assess- ment". The clinician is supported by a set of 16 questions (Cultural Formulation Interview/CFI ${ }^{18-20}$ ) to elicit culture specific factors, an approach closer to cultural anthropology and qualitative research than earlier DSM models. The inclusion of a focus on "causal explanations relevant to clinical practice" integrates the important concept of subjective health belief models that have been a standard in cultural anthropology as an aspect of the subjective illness experience.

The "cultural concepts of distress" and "cultural idioms of distress", finally take up the "idiom of distress" concept used in transcultural psychiatry for some time ${ }^{21,22}$ to describe the specific aspects of experiencing, expressing and describing distress in a culture. This aspect appears to be a crucial addition also for assessment as it addresses the frequent criticism of a too uncritical application of the narrow DSM PTSD concept to other cultures and might be equally or even more relevant than PTSD as part of the evidence of torture and in the recognition and documentation of disability and suffering.

\subsubsection{V codes}

$\mathrm{V}$ codes are important in forensic assessment as they create a further link to causality. They were already mostly included, but in practice not utilised sufficiently in ICD-10 CM. ${ }^{23}$ They offer a tool to code events such as imprisonment, torture, or persecution as the reason for observed symptoms or a diagnosis as requested in most forensic settings.

\section{Conclusion}

In spite of the caveats to be considered regarding a diagnostical level of assessment in general, DSM-5 has included a number of significant changes that can improve assessment of torture survivors if properly 
Table 2: Selected relevant V codes

V62.89 (Z65.4) Victim of Terrorism or Torture

V62.22 (Z65.5) Exposure to Disaster, War, or Other Hostilities

V62.5 (Z65.1) Imprisonment or Other Incarceration

V62.4 (Z60.3) Acculturation Difficulty

V62.4 (Z60.5) Target of (Perceived) Adverse Discrimination or Persecution

V63.9 (Z75.3) Unavailability or Inaccessibility of Health Care Facilities

\section{A short case example}

A 46-year old African refugee who had been tortured in his home country, applied for asylum in a European country, but his application was rejected. One of the reasons given was that during the psychological assessment, he attributed his psychological symptoms to spirits. During an appeal case, a thorough assessment based on the Istanbul Protocol, using DSM-5 criteria yielded a diagnosis of PTSD, and of a culture based idiom of distress that includes seeing the ghosts of others killed in war and in this case in prison. Further assessment by Magnetic
Resonance Imaging (MRI) also confirmed reported blunt brain trauma. In the conclusion of the findings, the symptoms were described as characteristic of the torture reported and were further linked to a Z65.4 code ("Victim of Torture"). The refugee received asylum based on the new report and kept the report in the hope of a later chance to use it in a court against the perpetrators. He was given the opportunity to pass on the report to the UN Special Rapporteur on Torture for monitoring purposes, but did not agree due to fear of reprisals. used. Some issues, such as aspects of mild brain injuries or a category similar to the ICD-10 F 62.0 category of "Enduring personality change after catastrophic experience", have not been or only partially implemented. The integration of well-established assessment tools such as the Cultural Formulation Interview (CFI) for culturally specific aspects, the inclusion of "idioms of distress" or the WHODAS for impairment and disability can further be seen as an improvement and are of major relevance in assessment of torture survivors. It remains to be seen how the upcoming ICD-11 and new dimensional diagnostic models will take up the relevant changes.

The need of adaptation and revalidation of important instruments such as the Clinician Administered PTSD Schedule (CAPS) or other standard diagnostic instruments can on the other hand be seen as a major challenge in this context. It might further be argued that the handbook should be available on an open platform, reflecting present standards in open publishing that underline the "access to health" concept and implicitly the access to diagnostic and research standards as a right that is also open to poor countries. 


\section{Acknowledgements}

With support from EFI "Human Rights in

Healthcare", University Erlangen-Nürnberg.

\section{References}

1. International Forensic Expert Group, Alempijevic D, Beriashvili R, Beynon J, Duque M, Duterte P, et al. Statement on access to relevant medical and other health records and relevant legal records for forensic medical evaluations of alleged torture and other cruel, inhuman or degrading treatment or punishment. J Forensic Leg Med. 2013 Apr;20(3):158-63. PubMed PMID: 23472795.

2. Perera C, Verghese A. Implementation of Istanbul Protocol for effective documentation of torture - review of Sri Lankan perspectives. J Forensic Leg Med. 2011 Jan;18(1):1-5. PubMed PMID: 21216370.

3. Furtmayr H, Frewer A. Documentation of torture and the Istanbul Protocol: applied medical ethics. Med Health Care Philos. 2010 Aug;13(3):279-86. PubMed PMID: 20419473.

4. Fincanci SK. The role of jurisdiction on persistence of torture in Turkey and public reflections. Torture. 2008;18(1):51-5. PubMed PMID: 19289882.

5. Iacopino V, Ozkalipci O, Schlar C. The Istanbul Protocol: international standards for the effective investigation and documentation of torture and ill treatment. Lancet. 1999 Sep 25;354(9184):1117. PubMed PMID: 10509518.

6. DSM-5 Task Force. Diagnostic and statistical manual of mental disorders: DSM-5. 5th ed. Washington, D.C.: American Psychiatric Association; 2013. xliv, 947 p. p.

7. Mollica RF, Caspi-Yavin Y, Bollini P, Truong T, Tor S, Lavelle J. The Harvard Trauma Questionnaire. Validating a cross-cultural instrument for measuring torture, trauma, and posttraumatic stress disorder in Indochinese refugees. J Nerv Ment Dis. 1992 Feb;180(2):111-6. PubMed PMID: 1737972.

8. Malekzai AS, Niazi JM, Paige SR, Hendricks SE, Fitzpatrick D, Leuschen MP, et al. Modification of CAPS- 1 for diagnosis of PTSD in Afghan refugees. J Trauma Stress. 1996 Oct;9(4):891-8. PubMed PMID: 8902755.
9. Blake DD, Weathers FW, Nagy LM, Kaloupek DG, Gusman FD, Charney DS, et al. The development of a Clinician-Administered PTSD Scale. J Trauma Stress. 1995 Jan;8(1):75-90. PubMed PMID: 7712061.

10. Pope KS. Psychological assessment of torture survivors: Essential steps, avoidable errors, and helpful resources. Int J Law Psychiatry. 2012 Sep;35(5-6):418-26. PubMed PMID: 23040707.

11. Wenzel T. Torture. Curr Opin Psychiatry. 2007 Sep;20(5):491-6. PubMed PMID: 17762594.

12. Wenzel T, Griengl H, Stompe T, Mirzaei S, Kieffer W. Psychological disorders in survivors of torture: exhaustion, impairment and depression. Psychopathology. 2000 Nov-Dec;33(6):292-6. PubMed PMID: 11060511.

13. Kalt A, Hossain M, Kiss L, Zimmerman C. Asylum seekers, violence and health: a systematic review of research in high-income host countries. Am J Public Health. 2013 Mar;103(3):e30-42. PubMed PMID: 23327250.

14. McColl H, Higson-Smith C, Gjerding S, Omar MH, Rahman BA, Hamed M, et al. Rehabilitation of torture survivors in five countries: common themes and challenges. Int J Ment Health Syst. 2010 Jun 18;4:16.PubMed PMID: 20565852.

15. World Health Organization. WHO psychiatric disability assessment schedule (WHO/DAS), with a guide to its use. Geneva: World Health Organization; 1988. viii, 88 p. p.

16. den Otter JJ, Smit Y, dela Cruz LB, Ozkalipci O, Oral R. Documentation of torture and cruel, inhuman or degrading treatment of children: A review of existing guidelines and tools. Forensic Sci Int. 2013 Jan 10;224(1-3):27-32. PubMed PMID: 23199437.

17. Keatley E, Ashman T, Im B, Rasmussen A. Selfreported head injury among refugee survivors of torture. J Head Trauma Rehabil. 2013 NovDec;28(6):E8-E13. PubMed PMID: 23348404.

18. Aggarwal NK, Nicasio AV, DeSilva R, Boiler M, Lewis-Fernandez R. Barriers to implementing the DSM-5 cultural formulation interview: 
a qualitative study. Cult Med Psychiatry. 2013 Sep;37(3):505-33. PubMed PMID: 23836098.

19. Lewis-Fernandez R, Aggarwal NK. Culture and psychiatric diagnosis. Adv Psychosom Med. 2013;33:15-30. PubMed PMID: 23816860.

20. Mezzich JE, Caracci G, Fabrega H, Jr., Kirmayer LJ. Cultural formulation guidelines. Transcult Psychiatry. 2009 Sep;46(3):383-405. PubMed PMID: 19837778.

21. Parsons CD, Wakeley P. Idioms of distress: somatic responses to distress in everyday life. Cult
Med Psychiatry. 1991 Mar;15(1):111-32. PubMed PMID: 2060312.

22. Nichter M. Idioms of distress: alternatives in the expression of psychosocial distress: a case study from South India. Cult Med Psychiatry. 1981 Dec;5(4):379-408. PubMed PMID: 7326955.

23. Prophet S. V codes: supplementary classification of factors influencing health status and contact with health services. J AHIMA. 1996 Jun;67(6):16-8, 20, 22; quiz 4-5. PubMed PMID: 10157903. 